\title{
El octubre chileno: voces y luchas feministas
}

\author{
Maria Vanesa Stevani Gisletti \\ Universidad de Valparaiso, Chile \\ vanesastevanig@gmail.com \\ Claudia Montero \\ Universidad de Valparaiso, Chile \\ claudia.montero@uv.cl
}

\section{1. ¡ARriba las QUe LUChan!}

\section{María Vanesa StevaniGisletti}

Universidad de Valparaíso - Coordinadora feminista 8 de marzo de Valparaíso, Chile. vanesastevanig@gmail.com

El 18 de octubre del 2019, Chile se remeció de un largo letargo, a veces interrumpido por alguna que otra manifestación que exigía reformas a una estructura económico- social imposible de permear.

Pasaron años, décadas, y nada cambió: "La alegría no llegó”. Por el contrario, los gobiernos pos-dictadura agudizaron las desigualdades sociales, consolidaron un modelo económico legado de la última dictadura cívico-militar de Pinochet que estableció una clase político-empresarial que sistemáticamente ha saqueado al territorio y a sus habitantes por medio de privatizaciones de los servicios básicos, imponiendo la flexibilidad laboral, precarizando los trabajos, manteniendo sueldo y pensiones miserables que en promedio no llegan a los 200 dólares mensuales, financiando con el erario público el $60 \%$ de un sistema de salud privado que solo atiende al $15 \%$ de la población, mientras que la mayoría muere en las salas de urgencia de hospitales o engrosan largas listas de espera que durante años pacientemente deben aguardar para tratarse u operarse. Una justicia ciega ante la colusión de grandes empresas que -por largo tiempo- mantienen los precios de los medicamentos y alimentos a precios exorbitantes y, cuando son denunciados, después de negociar a costa del pueblo, son "castigados" obligándolos a ir a clases de moral: sin pagar un peso, sin un día de cárcel. Ese mismo modelo nos ha dejado con una educación pública que recibe ataques permanentes porque es la única que denuncia la falta de insumos y financiamiento para estudiantes que dependen de ella para educarse o alimentarse. Con territorios con sequías por la privatización de sus napas de agua pues las grandes empresas desvían cursos enteros de ríos y lagos dejando a la población sin este recurso indispensable para la vida. Estas son las reales condiciones en las que vive la población. "No fueron 30 pesos, fueron 30 años": los y las estudiantes dieron la muestra de rebeldía, una vez más, demostrando que la evasión del metro era la forma de decir basta, a un modelo neoliberal que para el mundo era el milagro chileno, pero que por dentro se desangra, colapsa, que no es más que una estructura que debía ser permeada por la voz de la gente.

El descontento silencioso se transforma en gritos, canticos, cacerolazos, marchas multitudinarias con pancartas, con banderas. Ocupa regiones, ciudades, barrios, en todo el país llenos de pena, bronca y, también, de alegría de saber que no son pocos, sino miles, millones que exigen un cambio. Este movimiento social levanta consignas: "Hasta que la dignidad se haga costumbre”, "El pueblo unido jamás será vencido", "Chile Despertó”. La gente se cansó y la olla estalló, fue mucha la presión. Es mucha miseria económica, política y social, ante una clase dirigente sorda, distante, que no vio venir esta ola de conflictos, porque su alejamiento social e inoperancia política no la dejó ver lo evidente, lo real, la cotidianeidad. Una clase dirigente que quedó 
atónita al ver que las plazas públicas eran colmadas por ciudadanos y ciudadanas que exigían un cambio total, que decidían querer vivir con "Dignidad", con cacerolas en mano. Cada rincón de Chile se organizó en ollas comunes, asambleas ciudadanas, la rutina desapareció, levantamos la cara y empezamos a reconocernos, a mirarnos a saber que no estábamos solos, sino que éramos miles que decíamos basta. Cada protesta se ha transformado en un espacio de debate, de diálogo ciudadano, de levantar propuestas sociales y hacer una agenda donde todos y todas somos partícipes de lo que necesitamos y queremos para nuestras familias y territorios.

Hace un mes y algunos días que estoy con el corazón apretado, escucho sirenas de bomberos, patrulleros, aviones que sobrevuelan casas y poblaciones. Durante 30 días hemos salido a las calles, nuestra voz se ha escuchado:“¡De Norte a sur, de Este a Oeste, daremos la pelea, cueste lo que cueste...!”, ha sido hasta ahora un costo muy alto: 26 muertos; más de 200 personas con daños oculares por lanzamientos de balines de las fuerzas represivas; 3 casos donde han perdido en su totalidad su visión; 93 casos de violencia político sexual contra mujeres niñas, niños y disidencias sexuales; 2.670 denuncias por violación a los derechos humanos, la feroz represión empleada por carabineros, PDI y Militares. La contestación del gobierno fue represión al pueblo, acallar por la fuerza, deslegitimar las manifestaciones infiltrando policías y militares que quemaron tiendas y supermercados permitiendo el saqueo e incendios del metro y el retail, para luego mostrar en los medios su desaprobación, legitimando su accionar violento.

Las mujeres estamos desde hace mucho tiempo presentes en varios escenarios de la vida denunciando la destrucción de bosques, la crisis del modelo extractivista, la violencia de género, las desigualdades laborales, la violencia obstétrica,abogamos por un aborto legal, libre y gratuito, exigimos una educación no sexista, luchamos por la valorización del trabajo doméstico formal e informal remunerado, por el resguardo a la crianza protección de la infancia, por una ley de hogares monoparentales, por proyecto de crianza y maternidad para mujeres privadas de libertad, por la destrucción de un sistema capitalista y patriarcal que es una alianza criminal que nos desplaza e invisibiliza los saberes, creencias y prácticas feministas. Las mujeres tenemos mucho que aportar instalando un nuevo tejido social humano, equitativo, respetuoso de las diversidades y diferencias, creando oportunidades en estas rebeliones sociales donde las mujeres estamos presentes, organizando conversatorios, creando asambleas o cabildos feministas desde donde levantamos, mostramos y practicamos nuestra sororidad. Estamos marchando codo a codo con todo el territorio, donde nuestras miradas son compartidas, aceptadas, revisadas, debatidas. Desde diversos colectivos feministas, nos apañamos, protegemos y mostramos nuestra lucha gritando:

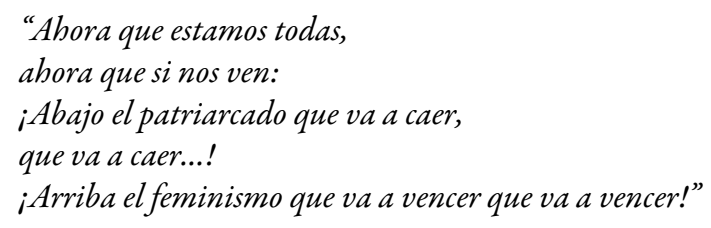

Las mujeres, diversidades y disidencias sexuales nos hacemos eco de este canto, en la lucha que cada día redoblamos, al sustentar nuestros hogares, al organizar ferias libres feministas para que varias compañeras sin trabajo puedan ofrecer sus productos a la comunidad. Denunciamos los casos de violencia política -sexual que durante estas manifestaciones han sufrido compañeras y compañeres a manos de militares y carabineros de Chile: "El estado opresor es el macho violador"

Salimos a las calles a luchar contra fuerzas policiales que detienen arbitrariamente a manifestantes (especialmente a mujeres, disidencias y diversidades) que son violadxs y torturadxs en carros policiales o en centros clandestinos sin pasar por la detención formal en comisarías, para luego ser dejadxs en la vía pública a las 24 o 48 horas posteriores a su detención con heridas físicas y graves daños mentales y emocionales provocados por un estado que castiga, reprime y trata de todas las maneras posibles de acallar nuestra lucha. 
En los últimos años, el mensaje ha sido claro para todas aquellas mujeres que fueron a incomodar con denuncias, visibilizando problemas en sus territoritos, ríos, bosques, poblaciones, sequías, negociados de grupos de poder, empresariales y comunicacionales: esas mujeres fueron asesinadas, amenazadas, encarceladas, torturadas -como los casos de Macarena Valdés y Nicolasa Quintreman. El estado ha implementado una persecución y asesinato de líderes sociales. Su muerte es nuestra bandera de lucha. ¡No dejaremos bajo el manto de la impunidad a sus victimarios! Desgraciadamente estas prácticas se han replicado en los últimos días. Hace solo una semana,una fotógrafa ha sido asesinada, Albertina Martínez Burgos. Ella como muchas otras mujeres, ponía su lente en el foco de la represión, de la lacrimógena, en el balín que hiere, en los golpes y detenciones. Llevaba un registro visual de la realidad permanente que vivimos en la capital y regiones, su cámara mostraba lo que los que los medios de comunicación serviles a las autoridades y grupos empresariales no muestran, no escuchan, ni se debate y, si lo hacen, es desde la mirada reprobatoria como si los que saliésemos a las calles no fuéramos miles de ciudadanas, sino, simples delincuentes pertenecientes a grupos aislados que no quieren escuchar las propuestas salvadoras de un gobierno que se cae a pedazos, que sigue desconociendo el liderazgo femenino en la sociedad, que solo habla de dar una mayor cuota de cupos políticos pero que jamás ha dialogado para conocer siquiera nuestras demandas.

En lo que va del año, en Chile hay 41 mujeres asesinadas por sus convivientes o ex parejas, según la ley que contempla los femicidios. La violencia contra la mujer por otros hombres o agentes del estado no es tomada como tal, sino como simples asesinatos. Si tenemos en cuenta la cantidad de mujeres violentadas por hombres que no tuvieron una relación sentimental, el número se eleva a 98 asesinadas por machitos represores. Permanentemente nos debemos cuidar, saber si llegamos a nuestras casas o quedarnos en lugares de acogida porque los seguimientos y secuestros están a la orden del día.

Ayer y hoy las mujeres levantamos nuestra voz, nuestro puño, caminamos juntas y organizadas. Sabemos que en momentos de nuestra historia mundial y nacional han querido quebrarnos, doblegarnos, silenciarnos, a través del uso de todos los métodos característicos de estados fascistas o dictatoriales. Pero una vez, más fortalecidas y conscientes, tenemos la oportunidad de terminar con este sistema capitalista- patriarcal, de reunirnos para construir, de organizar cooperativas de trabajo, de hacer redes de comercio local, de unificar nuestras demandas feministas para un nuevo tejido social, podemos crear una política desde la empatía y las lógicas horizontales dejando de lado los proyectos individuales y priorizar los colectivos, consolidando la autoformación territorial y local, dando paso al trabajo solidario, colectivo y circular donde podamos desarrollarnos, construyendo un territorio respetado por todxs, favoreciendo el encuentro, la reflexión colectiva y crítica, la creatividad y/o la autoformación, generando una verdadera comunidad.

Por ello, como feministas, rechazamos los acuerdos de paz realizados entre cuatro paredes, entre gallos y medianoches, bajo la misma lógica segregacionista y dominante, con representantes de partidos políticos totalmente deslegitimados que no han cedido un ápice frente a la nueva agenda social levantada en las calles, que pretenden acallar nuestras voces creando un plebiscito que no da cabida a los y las verdaderas ciudadanas que exigimos una asamblea constituyente para instalar las demandas y construir una constitución que represente a toda la sociedad. Además, "sin justicia no hay paz", exigimos la inmediata aclaración, reparación y castigo por la violación de los derechos humanos y la violencia política sexual ejercida por parte de las fuerzas especiales de carabineros y fuerzas armadas. Por ello, las feministas seguiremos en todos los ámbitos marcando nuestro paso en las calles, en la academia, en los espacios laborales, en los hogares, en cada rincón, para que se asiente la construcción de una sociedad respetuosa de las diferencias e igualitaria en sus derechos. Por este fin, seguiremos dando pelea a los poderes que no quieren renunciar a sus prerrogativas y privilegios y los organismos internacionales que los apoyan y aplauden su accionar y métodos represivos. La nuestra es una lucha por nuestra vida, porque hace 30 años nos están aniquilando. Hoy, esta declaración de guerra que han hecho Piñera y los políticos expresa claramente su voluntad de exterminio de un pueblo que claramente se levantó por su libertad y dignidad. Escribo al calor de esta lucha, que sabemos cuándo y por qué empezó pero aún no cesa, nos nutrimos de la fuerza y el canto a coro por las calles: 


\author{
iArriba las que luchan! \\ iAbajo el fascismo! \\ iArriba el Feminismo!
}

\title{
2. LASTESIS Y "UN VIOLADOR EN TU CAMINO"1
}

\author{
Claudia Montero \\ Universidad de Valparaíso \\ claudia.montero@uv.cl
}

El 18 de octubre de 2019 marcó un momento de cambio en el Chile contemporáneo. Ya es conocido el hecho del desate de una revuelta social sin precedentes desde el fin de la dictadura cívico-militar (1989). Una revuelta en el país que se había alzado como el ejemplo "exitoso" del neoliberalismo, el paraíso de la sociedad de libre mercado, de la felicidad a crédito y de una sociedad sin conflicto. Como dice el lema de las movilizaciones "Chile despertó". La revuelta encabezada por estudiantes, movilizó a diversos sectores sociales para destapar todas las tristes verdades de injusticias que ya se hacían crónicas. El movimiento feminista sostuvo más alta la bandera de la condena al patriarcado neoliberal, ese que carga a las mujeres con la tarea de sostener ese supuesto éxito con el trabajo feminizado. Trabajo que no importa el nivel en el que se desarrolle, no es reconocido o desigualmente pagado. En medio del estallido, a poco más de un mes, se articularon diversas expresiones de protesta FUEGO, Acciones en Cemento, el arte fuera de la sala, entre las que se presentó la performance del colectivo de Valparaíso Lastesis, "Un violador en tu camino". Lastesis haciendo honor al feminismo, dictaron cátedra de cómo analizar, interpelar, acusar y demandar al patriarcado con un mensaje simple y sin dejar herides o muertes.

"El octubre chileno" responde a más de 70 años de instalación del neoliberalismo en Chile. Con unos primeros intentos en los años 50 (contratación de la misión Klein Sacks y la formación de los "Chicago Boys") y su consagración con la dictadura cívico-militar, que la cimentó en la Constitución de 1980. Su éxito radicó en que logró implementar las políticas económicas de achicar el estado (reduciendo el gasto social) y avalar al mercado como regulador de las decisiones sociales (política de privatización), todo ello, sin contrapeso social, neutralizado por la represión política de la dictadura. En concreto, los cambios se tradujeron en la privatización de los servicios como la previsión social, el sistema de salud y de educación. Según consagra la Constitución, estos derechos dependían ahora de la capacidad personal de cada ciudadano/a, quien podía acceder a los servicios que desee, de acuerdo a su libertad para contratar lo que se ajuste a sus requerimientos. Quienes no tengan la capacidad de pago, podrían contar con los subsidios del estado. La subsidiaridad es el principio que sustenta la política de privatización de los servicios, y que quedó protegida legalmente en la Constitución de 1980. Esta hace que las personas sean las titulares de subsidios para pagar el servicio que requieran en la institución proveedora que prefieran. Con ello, se liquida el financiamiento basal de los servicios públicos y se los condena a la desaparición. Se suma a ello un sistema que permitía una acumulación para las empresas sin comparación en otros países, y dejando que murieran todas aquellas iniciativas que no pudieran sobrevivir en la competencia de la oferta y demanda.

La libertad de elegir enmascaraba el juego perverso de fortalecer a las personas para creer que el esfuerzo personal llevaría a una mejor calidad de vida. Promesa que por mucho que se trabajara nunca se lograba: el endeudamiento terminaba siendo la herramienta de acceso a los bienes/servicios. La vida en comunidad se perdía en el deseo individual de satisfacer las propias necesidades. Y la reproducción humana seguía sin ser considerada como un "gasto" en la planilla de egresos del sistema neoliberal.

Los gobiernos democráticos no rompieron con este modelo. La acumulación siguió sin cuestionarse a pesar de los indicadores de desigualdad que ponían a Chile con unos ingresos per cápita de país OCDE y con una distribución que alertaba el estallido social de este octubre, en la magnitud de terremoto grado 10. 
Les estudiantes estuvieron a la vanguardia demostrando el descontento frente a ese modelo: desde el año 2001 cuando hicieron el primer "mochilazo", luego con la movilización pingüina del 2006 y el paro histórico del 2011. Exigieron educación como un derecho y no como un bien de consumo y apuntaron al neoliberalismo como causante de desigualdades, falta de calidad y tumba de la educación pública. Le secundó el movimiento no Más AFP, contra las administradoras privadas de las pensiones, que cuestionó el principio de ahorro individual, demostrando además que los cotizantes nos son los dueños de su dinero (paradojas del liberalismo).

El movimiento feminista que aparentemente vivía un "nuevo silencio", en realidad se mantuvo en alerta y en la denuncia constante. El feminismo autónomo se mantuvo activo contra la violencia de género, los pactos de silencio, el saqueo de recursos naturales e hizo causa común con el pueblo mapuche, en una articulación de colectivos que estuvieron activos en medio de la anestesia del éxito económico de los años 90 (aquí tenemos colectivos como Las Clorindas, Mujeres contra la Globalización, Ayuquelén, entre otros). Hacia el 2000 distintes feministas comenzaron a articularse por la despenalización del aborto, que se consiguió en 2017, aunque sólo en tres causales (riesgo de vida de la madre, inviabilidad fetal y violación). En los últimos 20 años, el efecto de feministas ejerciendo en diversos ámbitos se hizo notar en la formación de una generación de mujeres jóvenes que protagonizaron todos los movimientos de demanda social. No extraña entonces el Mayo Feminista del 2018, con toda su fuerza desestabilizadora, que obligó a repensar relaciones personales e institucionales.

Todos los movimientos del 2000 fueron antecedente de este estallido social del que muchos ahora declaran saber que se vendría, aunque en realidad fue inesperado y sorprendió a todo el mundo, tanto por el momento como por su envergadura. La violencia estructural generada por la acumulación de un porcentaje ínfimo de la población, basada en la explotación de la mayoría, a la que además se le instala el deseo por el consumo (como si se perteneciera al mundo de los ricos, aunque en realidad era esclava de servicios sociales inexistentes), fue naturalizada al nivel de hacerse imperceptible por quienes creían en el modelo. En algunas pancartas de la protesta actual se leía que el milagro chileno no era que funcionara el modelo de mercado, sino que no se habían expresado las quejas.

La magnitud del despertar ha quedado en evidencia en la violencia desatada contra la manifestación. La clase dirigente ciega ante el malestar de la gente, ya que en este país tan desigual los ricos nunca se relacionan con el resto, salvo en condiciones de subordinación, alejada de toda empatía, solo sintió molestia ante el reclamo. No comprendió y no entendió por qué la gente no quería vivir en el paraíso que ellos veían. Seguro porque su mirada alcanzaba a tocar la base, invisibilizando a los que están sosteniéndolos. Frente a la rabia acumulada, sólo respondieron con violencia.

En el primer fin de semana hubo muertos. A los tres días, se respondió con estado de emergencia y el gobierno nos hizo recordar que la dictadura fue cívica también, porque son los mismos civiles golpistas que ahora vuelven a aplicar lo practicado a partir del 73: toque de queda, detenciones ilegales, desapariciones, torturas y violencia sexual. A la alegría por el estallido que nos hizo sentir vives, que la comunidad no estaba perdida, se le sumó la angustia, el dolor y la rabia por otro capítulo de violaciones a los Derechos Humanos.

El movimiento feminista que salió a las calles a protestar, también condenó esta violencia y particularmente el ensañamiento contra las mujeres, que, probado está, frente a una misma situación de detención, hombres y mujeres han recibido trato diferenciado. Las mujeres y personas de las disidencias han sido violentadas sexualmente en su mayoría. ¿Por qué se castiga especialmente a les hijes rebeldes de este Chile despierto?

Lo más seguro es que los perpetradores tenían en la memoria el "Mayo Feminista 2018", movimiento de carácter global, pero que en Chile tuvo una dimensión, de nuevo, que nadie pudo prever. Fue una nueva ola feminista que recuperó la memoria de un movimiento de mujeres activo constantemente desde el inicio del siglo XX. Que recogió la experiencia del Movimiento Pro Emancipación de la Mujer Chilena (MEMCH) que hizo sistemática la salida a la calle del feminismo en los años 30. Aunque tuvo que sortear el silenciamiento de la historia que ha tratado de excluir a las mujeres del relato. A pesar del ocultamiento, 
cada nueva generación de feministas ha debido hacer un esfuerzo de recuperación de su propia historia y la del movimiento de mujeres.

En esa recuperación histórica y reconstrucción de genealogías, esta nueva ola confirma que el feminismo nunca ha sido silente frente a la injustica. Ha habido una constante demanda por una sociedad con derechos para todes, por la emancipación de las mujeres; por la denuncia a una sociedad basada en jerarquías de género y sexuales que no se hace cargo de las políticas de cuidado, dejando a mujeres y disidentes cumpliendo tareas invisibilizadas y que son la base de la acumulación de otros.

El 25 de noviembre de 2019, entre marcha y protesta, el feminismo chileno salió a la calle una vez más a condenar la violencia contra las mujeres. En un ejemplo de protesta, diversas manifestaciones, ritos y marchas pacíficas se repitieron a lo largo del país (ejemplo de ello es el Rito a la Calle en Valparaíso y la Marcha del Silencio que se hace cada mes). Entre ellas, se presentó en Valparaíso por segunda vez en menos de 10 días, la performance del Colectivo Lastesis "Un violador en tu camino".

La propuesta de Lastesis es simple y efectiva. Podemos entenderla como una síntesis de 100 años de movimiento feminista en Chile. Logra, en una puesta en escena masiva, recoger la teoría feminista, traducirla en un mensaje, analizar la realidad y denunciar. Todo en un paquete de no más de tres minutos que interpela a la sociedad completa en sus distintos niveles sin dejar ningún herido/a, administrando la tecnología para lograr eficacia al apuntar directamente al poder con el dedo.

Lastesis es un colectivo feminista compuesto por cuatro mujeres de Valparaíso, con formación académica en diversas disciplinas de humanidades y las artes. No es casual que sean porteñas (como se llama a les habitantes de Valparaíso en Chile), ya que esta ciudad, por su propia historia urbana, comercial y emancipada, vio nacer a grupos pioneros en la defensa de derechos ya a fines del siglo XIX. De esta ciudad son las primeras organizaciones de mujeres tanto obreras como republicanas, acá nacieron algunos de los periódicos de y para mujeres, entre otras muchas manifestaciones libertarias.

Personalmente, vi por primera vez a Lastesis en la presentación de Silvia Federici en el Teatro Municipal de Valparaíso, a fin de 2018. En esa ocasión la performance presentaba una lectura del libro El Calibán y la Bruja. Traducía en lenguaje escénico los planteamientos teóricos de la autora, de forma que se podía observar la densidad de las ideas en un cuadro plástico donde cada elemento -el vestuario, el texto y la coreografíaexplicaban la explotación de las mujeres como base del sistema capitalista.

En la performance "Un violador en tu camino", realizan un ejercicio en el mismo tono, para ser presentado en la protesta callejera. Tal como lo han hecho mujeres de generaciones anteriores, supieron administrar los medios y formatos comunicativos con los que cuentan, para rasguñar la exclusión del espacio público y colar la propuesta feminista. En este caso, se sirvieron de las redes sociales para en un primer momento difundir la primera presentación realizada en Valparaíso el 11 de noviembre, y luego convocar a las presentaciones siguientes. De allí en adelante, el resto de las feministas de Chile y del mundo se autoconvocaron para protestar a través de la performance.

El estímulo inicial de "Un violador en tu camino" fue la muestra programada y difundida a través de Instagram de una serie de intervenciones artísticas en la calle a propósito de la protesta: FUEGO, acciones de Cemento, el arte fuera de la sala. Era una semana de intervenciones, varias de ellas en la Plaza Aníbal Pinto de Valparaíso, lugar neurálgico de la ciudad. Lastesis fueron convocadas para el día 20 de noviembre y ellas hicieron un llamado a su red de mujeres y disidencias para que las apoyaran con la primera muestra. Hubo un ensayo en los días previos..., y listo. Una vez presentada esta primera intervención, sus videos viralizados sirvieron de tutorial para todas aquellas que se sintieron convocadas a denunciar la violencia contra las mujeres en tanto en el contexto de protesta como en la vida general.

La letra de "Un violador en tu camino" tiene tres partes con sus respectivas contestaciones. En la primera, se recoge la teoría feminista que denuncia la subordinación que sufren las mujeres por su condición de sexogénero. Usa los tiempos verbales: "El violador eres tú/eras tú", y con ello muestra los distintos niveles en los que opera la exclusión de género. Por una parte es histórica, entonces, se ve en el pasado. Sin embargo, en la 
medida en que se mantiene la desigualdad, sigue operando y las mujeres seguimos sufriendo la opresión en el presente. Con el juego de las oraciones "Es la violencia que no ves/ya ves", denuncian la naturalización de la violencia de género a través de prácticas cotidianas como el micromachismo, que quedan al descubierto con la denuncia de la propia acción performática. No se ve la violencia porque se invisibiliza, pero ya "la ves" en el gesto feminista de denuncia constante, "ya la ves" porque Lastesis la muestra con esta acción de arte/ denuncia.

La letra incluye partes de acusación directa, que muestran que el sistema patriarcal está encarnado en todas las instituciones que, en su conjunto, oprimen a las mujeres. Es el estado y la familia, y en esta última, son todos los machos violadores. Esta es una metáfora significativa, ya que apela a todos los varones heterosexuales a revisar su relación de poder con las mujeres; a mirar la construcción de su masculinidad en función de cuánto o de qué forma violenta a las mujeres con las que se relacionan. Es esta misma apelación la que ha incomodado a tantos varones y la que ha llevado a reaccionar de forma burlesca (ejemplo de ello fue el equipo del Club América sub 17 de México). Volvemos a preguntarles entonces: ¿son todos los varones violadores?

La segunda parte denuncia la violencia que viven las mujeres en tanto mujeres y que, en el contexto de la represión del Octubre Chileno, fue ejercida contra las manifestantes detenidas. Esa denuncia se refuerza con la coreografía, en la que se realizan sentadillas. Este ejercicio es el símbolo de la expresión de poder en la jerarquía militar: quien está más arriba puede castigar a los subordinados obligándolos a hacer un número determinado de estas flexiones. Entre las denuncias de violaciones a los Derechos Humanos en las últimas protestas, se consignó este tipo de castigo a detenidos y detenidas, siendo especialmente vejatorias las ejercidas contra las mujeres.

Finalmente, la letra incluye un análisis del discurso del propio himno de Carabineros de Chile, que establece, sin ninguna metáfora, el lugar de las mujeres en la sociedad: pasiva, frágil y dispuesta al "amor". Todo lo contrario de una mujer feminista. De una mujer en la protesta y en la marcha. Todas ellas desobedientes del mandato patriarcal merecerían, según ese himno, el castigo por no quedarse tranquilas en su hogar.

La performance impactó de forma global. La denuncia, a pesar de que está contextualizada en Chile, es de alcance universal. El patriarcado amenaza con la violación a todas las mujeres del mundo, no importa su raza, clase o nivel educativo. Y cada mujer ha tenido alguna experiencia de violencia sexual en su vida. Por eso es tan asible para las mujeres, por eso es que se apropia y cobra nueva vida, al volver a circular por las redes sociales con la intervención personal contestando a la máxima: "Y la culpa no era mía, ni dónde estaba, ni cómo vestía", "Y la culpa no era mía: tenía X años; ni dónde estaba: en mi casa/colegio/trabajo/X, fue mi tío/cura/profesor XXX; ni cómo vestía: buzo deportivo, jeans, vestido...”.

Esta performance y sus reacciones nos recuerdan que el patriarcado está más vigente que nunca, que la revolución tiene que ser feminista, sino, no es revolución; que los cambios sociales son con mujeres y disidencias, que la justicia no tiene medida y que el feminismo sigue siendo el movimiento social que, a partir de la liberación de las mujeres, plantea la emancipación de todes les seres humanos.

\section{Notas}

1 Agradezco las respuestas de Paula Cometa, integrante de Lastesis, quien aportó con los datos de la intervención "Un violador en tu camino". 